\title{
Image Analysis of Renal DCE-MRI for the Detection of Acute Renal Rejection
}

\author{
Ayman El-Baz ${ }^{1}$, Aly Farag ${ }^{1}$, Rachid Fahmi ${ }^{1}$, Seniha Yuksela ${ }^{1}$, \\ Mohamed A. El-Ghar ${ }^{2}$, and Tarek Eldiasty ${ }^{2}$ \\ ${ }^{1}$ CVIP Laboratory, University of Louisville, Louisville, Kentucky, USA. \\ ${ }^{2}$ Urology and Nephrology Department, University of Mansoura, Mansoura, Egypt.
}

\begin{abstract}
Acute rejection is the most common reason of graft failure after kidney transplantation, and early detection is crucial to survive the transplanted kidney function. In this paper we introduce a new approach for the automatic classification of normal and acute rejection transplants from Dynamic Contrast Enhanced Magnetic Resonance Imaging (DCE-MRI). The proposed algorithm consists of three main steps; the first step isolates the kidney from the surrounding anatomical structures. In the second step, a novel nonrigid-registration algorithm is employed to account for the motion of the kidney due to patient breathing, and finally, the perfusion curves that show the transportation of the contrast agent into the tissue are obtained from the cortex and used in the classification of normal and acute rejection transplants. Applications of the proposed approach yield promising results.
\end{abstract}

\section{Introduction}

In the United States, approximately 12000 renal transplants are performed annually [1], and considering the limited supply of donor organs, every effort is made to salvage the transplanted kidney [2]. However, acute rejection - the immunological response of the human immune system to the foreign kidney - is the most important cause of graft failure after renal transplantation, and the differential diagnosis of acute transplant dysfunction remains a difficult clinical problem.

Currently, the diagnosis of rejection is done via biopsy, but biopsy has the downside effect of subjecting the patients to risks like bleeding and infections. Moreover, the relatively small needle biopsies may lead to over or underestimation of the extent of inflammation in the entire graft [3]. Therefore, a noninvasive and repeatable technique is not only helpful but also needed in the diagnosis of acute renal rejection. For this purpose, detection of acute renal rejection after kidney transplantations has become an on- going collaboration between the University of Mansoura and the CVIP Lab at the University of Louisville where Dynamic Contrast-Enhanced Magnetic Resonance Imaging (DCE-MRI) is applied prior to biopsy for its superior functional and anatomical information. In DCE-MRI, a contrast agent called Gd-DTPA is injected into the bloodstream, and as it perfuses into the organ, the kidneys are imaged rapidly and repeatedly. During the perfusion, Gd-DTPA causes a change in the relaxation times of the tissue and creates a contrast change in the images. As a result, the patterns of the contrast change gives functional information, while MRI provides good anatomical information which helps in distinguishing the diseases that affect different parts of the kidneys. However, even with an imaging technique like DCE-MRI, there are several problems; such as, (i) the spatial resolution of the dynamic MR images is low due to fast scanning, (ii) the images suffer from the motion induced by the breathing patient which necessitates advanced registration techniques, (iii) the intensity of the kidney changes non-uniformly as the contrast agent perfuse into the cortex which complicates the segmentation procedures.

To the best of our knowledge, there has been limited work on the dynamic MRI to overcome the problems of registration and segmentation. For the registration problem, Gerig et al.[4] proposed using Hough transform to register the edges in an image to the edges of a mask and Giele et al.[5] introduced a phase difference movement detection method to correct kidney displacements. Both of these studies required building a mask manually by drawing the kidney contour on a 2D DCE-MRI image, followed by the registration of the time frames to this mask.

\section{METHODS}

In this paper we introduce a novel and automated technique (i) to segment the kidney and (ii) to correct for the motion artifacts caused by breathing and patient motion; the details of which are given below. 


\section{$2.1 \quad$ Segmentation}

The first step of the proposed approach is to extract the kidney tissues from DCE-MRI's as shown in Fig. 1. Segmentation algorithms are based on using the deformable model guided by a stochastic force which represents the intensity and shape prior of the kidney. Details of the algorithm are presented in [6].

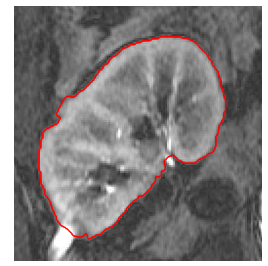

error $=0.3820 \%$

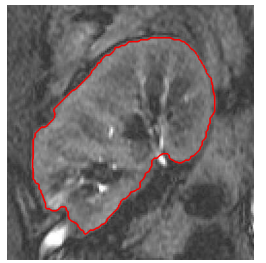

error $=0.3997 \%$
Figure 1. Segmentation results using the approach proposed in [6] with the errors w.r.t the radiologist segmentation.

\subsection{Model for the local deformation}

In DCE-MRI sequences, the registration problem arises because of the patient $\&$ breathing movements. To overcome this problem, we proposed a new a approach to handle the kidney motion. The propose approach is based on deforming the segmented kidney over closed equispaced contours (i.e. iso-contours) to closely match the prototype. We do not use a free-form deformation based on B-spline on square lattice because it requires additional smoothing constraints that lead to very time consuming computations. Instead we use evolution of the iso-contours guided with an exponential speed function in the directions minimizing distances between corresponding pixel pairs on the iso-contours of both the objects. The normalized crosscorrelation is used as image similarity measure insensitive to intensity changes (e.g. due to tissue motion in medical imagery and the contrast agent).

The first step of the proposed approach is to use the fast marching level set methods [7] to generate the distance map inside the kidney regions as shown in Fig. 2(a), (b). The second step is to use this distance map to generate equal space separated contours (iso-contours) as shown in Fig. 2(c) and (d). Note that the number of iso-contours depend on the accuracy and the speed which the user needs to achieve. The third step of the proposed approach is to use normalized cross correlation to find the correspondence between the iso-contours. Since we start with aligned images, we limit our searching space to a small window (e.g. $10 \times 10$ ) to improve the speed of the proposed approach. The final step is the evolution of the iso-contours; here, our goal is to deform the iso-contours in the first image (target image) to match the iso-contours in the second image (source image).
Before we discuss the details of the evolution algorithm lets define the following terminology:

- $\phi_{n_{i s o}}^{A}(h, \nu)$ is the iso-contours in the target image, where $h=1, \ldots, \mathcal{H}$ is the index of the control points in the given contour, $n_{\text {iso }}=1, \ldots N_{\text {iso }}$ is the index of the iso-contours, and $\nu$ is the iteration step.

- $\phi_{m_{i s o}}^{B}(\gamma)$ is the iso-contours in the source image, where $\gamma=1, \ldots, \Gamma$ is the index of the control points in the given contour, and $m_{\text {iso }}=1, \ldots, M_{\text {iso }}$ is the index of the iso-contours.

- $S$ is Euclidean distance between two corresponding points located on both iso-contours of both images.

- $S_{n_{i s o}, n_{i s o}-1}^{A}$ is the Euclidian distance between $\phi_{n_{\text {iso }}}^{A}(l, \nu)$ and $\phi_{n-1}^{A}(l, \nu)$

- $\mathrm{V}$ is the propagation speed function.

The most important step in the model propagation is the selection of the propagation speed function V. The selection of the speed function must satisfy the following conditions:

1. $\mathbf{V}=0$ if $S=0$

2. $\mathbf{V} \leq \min \left(S, S_{n_{i s o}, n_{i s o}-1}^{A}, S_{n_{i s o}, n_{i s o}+1}^{A}\right)$ if $S>0$; is the smoothness constraint, which prevents the current point from passing the closest neighbor contour as shown in Fig. 3.

The speed function of the following form satisfies the above conditions:

$$
\mathbf{V}=e^{\beta S}-1
$$

where $\beta$ is the propagation constant with the upper bound

$$
\beta \preceq \frac{\ln \left(\min \left(S, S_{n_{i s o}, n_{i s o}-1}^{A}, S_{n_{i s o}, n_{i s o}+1}^{A}\right)+1\right)}{S}
$$

Based on the speed function shown in Eq. (1) we can deform the iso-contours using the following equation as shown in Fig. 3(b):

$$
\phi^{A}(h, \nu+1)=\frac{\mathbf{V}}{S} \phi_{m_{i s o}}^{B}(\gamma)+\frac{S-\mathbf{V}}{S} \phi_{n_{i s o}}^{A}(h, \nu)
$$

for $h=1, \ldots, \mathcal{H}, m_{\text {iso }}=1, \ldots, M_{\text {iso }}, n_{\text {iso }}=$ $1, \ldots, N_{\text {iso }}$.

The final step of our approach is to segment the cortex from the segmented kidney. To achieve this task, we use the same approach but based only on the intensity. In Fig. 5, we show the cortex segmentation results on two of the kidneys. 


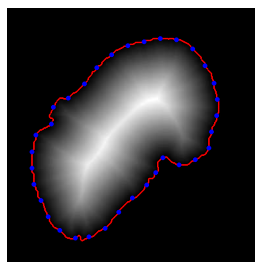

(a)

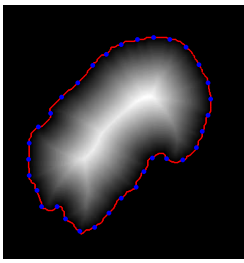

(b)
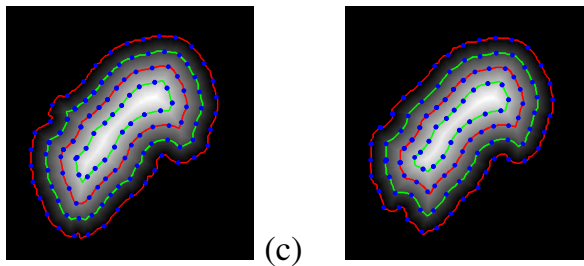

(c)

(d)

Figure 2. The distance map of two kidneys (a, b) and the isocontours (c, d).

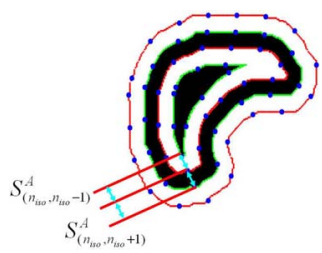

(a)

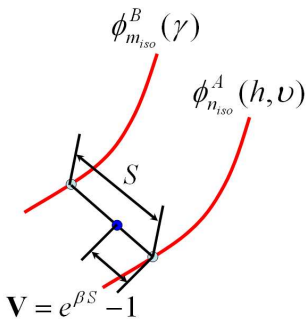

(b)

Figure 3. (a) Model constrains and (b) model evolution.

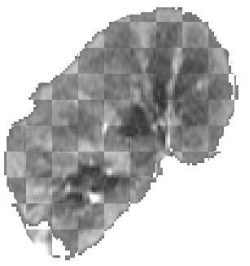

(a)

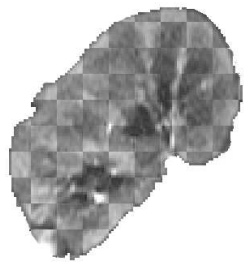

(b)

Figure 4. Checkerboard image to show the quality of the approach, (a) before non-rigid registration, and (b) after non-rigid registration.
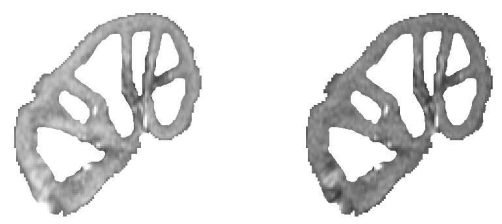

Figure 5. The segmentation of the cortex from the kidney images.

\subsection{Validation of our registration ap- proach using the F.E.M.}

In this section we propose to validate our deformable registration approach using the finite element (F.E.) method. Given a 2D image of the kidney, we simulate a deformation using a biomechanical modelling of the kidney tissue. The pair of images (deformed and non-deformed ones) is used to test our algorithm. The Abaqus/CAE (Ver. 6.5) ${ }^{1}$ environment was used to generate a cubic spline fit to the points representing the outer contour of the kidney object and then a 2D F.E. model was built from it. Figure 6(a), (b) and (c) show the 2D mesh before and after deformation, and the overlay of these two meshes, respectively. For the sake of generating a deformed shape only, we assumed the kidney tissue to be isotropic and homogeneous elastic material with a Young modulus $E=2500 \mathrm{~Pa}$ and a Poisson ratio $\nu=0.4$. Note that this model does not reflect the results of any rheological experiments conducted on the kidney tissue. A uniformly distributed pressure $P=100 \mathrm{~N}$ was applied normal to the boundary of the kidney. The points on this boundary are allowed to move freely in the $x$ and $y$ directions, but are constrained to rotate around the $z$ direction. The mesh consists of 1253 3-node linear plane stress angular elements. The average displacement of the induced deformation is $4.75 \mathrm{~mm}$, the minimum is $1.19 \mathrm{~mm}$, and the maximum is $6.9 \mathrm{~mm}$. The accuracy of the registration method is assessed by registering the simulated deformed image to the original one and comparing the recovered point displacements with the bio-mechanically simulated ones. The average registration error is about $1.54 \mathrm{~mm}$, with a maximum of $2.7458 \mathrm{~mm}$, a minimum of $0.0221 \mathrm{~mm}$, and a standard deviation of 0.5517 . This relatively proves the accuracy of our non-rigid registration technique used in this work.

\section{Results and Conclusion}

The ultimate goal of the proposed algorithms is to successfully construct a renogram (mean intensity signal curves) from the DCE-MRI sequences, showing the behavior of the kidney as the contrast agent perfuse into the transplant. In acute rejection patients, the DCE-MRI images show a delayed perfusion pattern and a reduced cortical enhancement. We tested the above algorithms on thirty patients; four of which are shown in Figure 7. The normal patient shows the expected abrupt increase to the higher signal intensities and the valley with a small slope. The acute rejection patients show a delay in reaching their peak signal intensities. From these observations, we have been able

\footnotetext{
${ }^{1}$ www.abaqus.com
} 
(a)

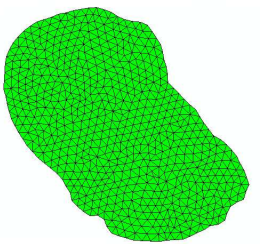

(c)

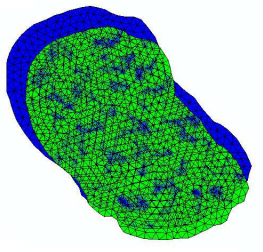

(d)

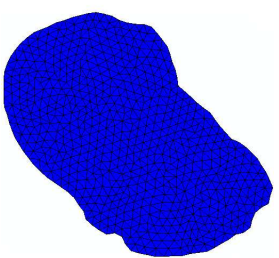

(b)
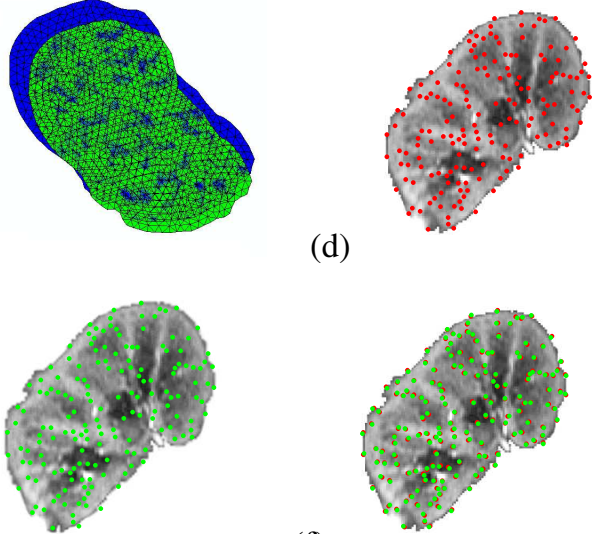

(e)

(f)

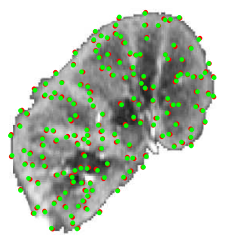

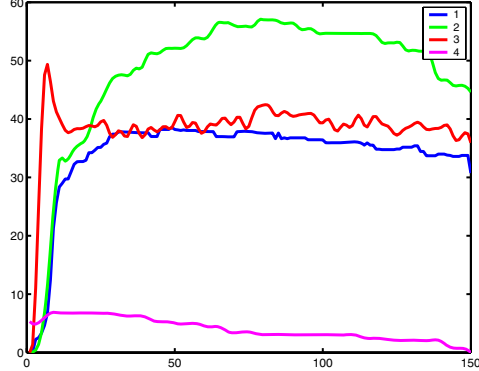

Figure 7. Normalized cortex signals from 4 subjects wrt. scan number. Subjects 1 and 2 are acute rejection, subject 3 is normal and subject 4 is chronic glomerulopathy proved by biopsy.
Figure 6. Validation of non-rigid registration. F.E mesh (a) before and (b) after deformation. (c) Meshes (a) \& (b) overlayed. (d)\&(e) Sample of the selected correspondence pairs determined from F. E meshes before \& after deformation. (f) Results of the proposed non-rigid registration approach.

to conclude that the relative peak signal intensity, time to peak signal intensity, the slope between the peak and the first minimum, and the slope between the peak and the signal measured from the last image in the sequence are the major four features in the renograms of the segmented kidney for classification.

To distinguish between normal and acute rejection, we use Bayesian supervised classifier learning statistical characteristics from a training set for the normal and acute rejection. The density estimation required in the Bayes classifier is performed for each feature by using a linear combination of Gaussians (LCG) with positive and negative components, their parameters are estimated using a modified EM algorithm which appeared in [?]. In our approach we used 50\% of the data for the training and the other $50 \%$ for testing. For testing data, the Bayes classifier succeeds to classify 13 out of 15 correctly ( $86.67 \%$ ). For the training data the Bayes classifier classifies all of them correctly, so the over all accuracy of the proposed approach is $93.3 \%$.

In this paper we presented a framework for the detection of acute renal rejection from Dynamic Contrast Enhanced Magnetic Resonance Images which includes segmentation of the kidneys from the abdomen images, non-rigid registration and Bayes classification.

\section{References}

[1] U.S. Department of Health and Human Services. Annual report of the U.S. scientific registry of transplant recipients and the organ procurement and transplantation network: transplant data: 1990-1999. Bureau of Health Resources Department, Richmond, VA; 2000.

[2] M. Neimatallah, Q. Dong, S. Schoenberg, K. Cho, and M. Prince, "Magnetic, "Magnetic resonance imaging in renal transplantation," J Magn Reson Imaging, vol. 10, no. 3, pp. 357-368, Sep 1999.

[3] D. Yang, Q. Ye, M. Williams, Y. Sun, T. C. C. Hu, D. S. Williams, J. M. F. Moura, and C. Ho.,"USPIO-Enhanced Dynamic MRI:Evaluation of Normal and Transplanted Rat Kidneys," Magn. Reson. in Medicine, vol. 46, 1152-1163, 2001.

[4] G. Gerig, R. Kikinis, W. Kuoni, G.K. van Schulthess, and O. Kubler,"Semiautomated ROI analysis in dynamic MRI studies: Part I: image analysis tools for automatic correction of organ displacements," IEEE Trans. Image Processing, vol. 11, no. 2,pp. 221-232, 1992.

[5] E. Giele, "Computer methods for semi-automatic MR renogram determination," Ph.D. dissertation, Department of Electrical Engineering, Eindhoven University of Technology, Eindhoven, 2002.

[6] A. El-Baz, S. E. Yuksel, A. A. Farag, H. Shi, T. ElDiasty, and M. Ghoneim, 2D and 3D Shape Based Segmentation Using Deformable Model, Proc. of International Conference on Medical Image Computing and Computer-Assisted Intervention, MICCAI-2005, Palm Springs, California, USA, October 26-29, 2005.

[7] J. A. Sethian, "Fast marching level set method for monotonically advancing fronts," Proc. Nat. Acad. Sci. USA, vol. 93, pp. 1591-1595, Feb. 1996. 